\title{
Patient, Surgeon, and Health Care Worker Safety during the COVID-19 Pandemic
}

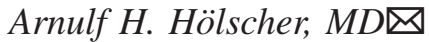

Keywords: COVID-19, health care workers, minimally invasive surgery, pandemic, patient safety, SARS-CoV-2, surgeon safety

(Ann Surg 2021;274:681-687)

\section{PATIENT SAFETY}

$T^{\mathrm{h}}$ he painting of Pablo Picasso from 1897 entitled "Ciencia y Caridad" describes 2 aspects of patient safety very well (Fig. 1). At the center of the tableau is a bedridden patient. The physician measures the pulse while looking gravely and concentratedly at his pocket watch. He represents in the interpretation of Picasso, whose uncle was a physician, the rational and scientific aspects of treatment. The nun looks toward the patient, serves fluid, and takes care of the patient's child. She stands in this painting from the century before last for the emotional and practical aspects of care. Both are important for the safety and wellbeing of our patients. The patient should feel secure and confident by virtue of receiving both empathic and scientifically grounded safe treatment under the maxim "primum nihil nocere."

"Patient safety is a framework of organized activities that creates cultures, processes, procedures, behaviors, technologies, and environment in health care that consistently and sustainably lower risks, reduce the occurrence of avoidable harm, make error less likely, and reduce its impact when it does occur." ${ }^{1}$ Undoubtedly securing patient safety now requires teamwork, but in the case of surgical treatment patient safety nevertheless remains a paramount issue for the surgeon, who will often have been chosen personally by the patient. ${ }^{2}$ The surgeon assumes responsibility for his patient and accompanies him during the pre, intra- and postoperative periods longer than all other contributing allied disciplines. This results in a special, close, and reciprocal relationship and the patient will ultimately see the surgeon as primarily responsible for the success or failure of the treatment as a whole.

\section{Burden of Impaired Patient Safety Before the COVID-19 Pandemic}

Many medical centers, health care systems, national and international organizations have made great efforts to analyses the frequency of adverse medical events and to determine their origins to establish best procedures and guidelines for their prevention. ${ }^{3-8}$ The central aims of these efforts were firstly to reduce the burden of morbidity and mortality borne by patients and their families and secondly to reduce both the enormous costs of subsequent treatment and litigation, and the wider economic consequences of disability and unemployment.

\footnotetext{
\arnulf.hoelscher@icloud.com.
}

The authors report no conflicts of interest.

Copyright (C) 2021 Wolters Kluwer Health, Inc. All rights reserved.

ISSN: 0003-4932/21/27405-0681

DOI: $10.1097 /$ SLA.0000000000005124
On average 1 in every 10 patients experiences an adverse event while receiving hospital care in high-income countries. ${ }^{1}$

The most frequently occurring adverse events are health careassociated infections, venous thromboembolism, adverse drug events, pressure injuries, diagnostic errors, and wrong site surgeries. ${ }^{3}$

In high-income countries up to $15 \%$ of hospital expenditure is spent on preventable safety failures. ${ }^{3}$ The WHO Regional Office for Europe reported in 2019 that $>750,000$ medical errors resulting in harm occur in Europe each year, including 260,000 incidents resulting in permanent disability and almost 100,000 incidents resulting in death; all of which leads to $>3.2$ million additional days of hospitalization annually.

Following the recognition of the enormous individual and societal burdens resulting from unsafe care, new efforts to tackle the problem were initiated. The European Surgical Association (ESA) played a central role in the 2020 European Patient Safety Policy Summit in Brussels. ${ }^{10}$ In a consensus statement 8 European associations under the auspices of the European Society of Anesthesiology urged the Commission of the European Union to:

ensure that minimum standards of care are met;

- facilitate data collection and analysis to measure, monitor, and improve patient safety and quality of care and;

promote excellence in surgical care and anesthesiology through education, research, and innovation.

In January 2021, the WHO initiated the "Global Patient Safety action plan 2021-2030" with the aim of maximally reducing avoidable harm due to unsafe health care practice around the globe ("zero harm"). ${ }^{1}$

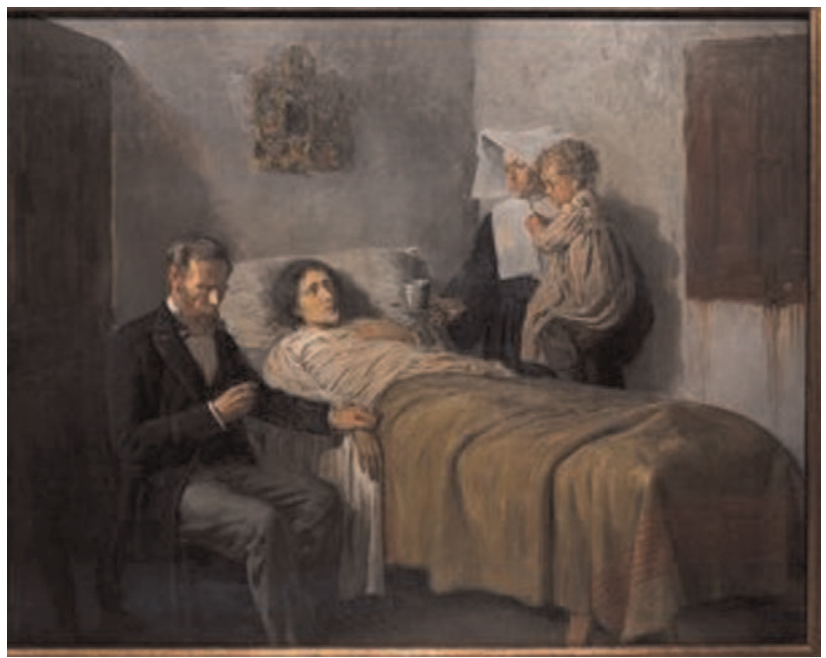

FIGURE 1. Painting "Ciencia y Caridad" of Pablo Picasso from 1897 (Museo Picasso Barcelona). 
These developments in patient safety were however overtaken by the unprecedented safety challenges that arose from the development of the COVID-19 global pandemic in 2020 .

\section{COVID-19 Pandemic and Patient Safety}

By June 1, 2021 the WHO Coronavirus (COVID-19) Dashboard was reporting nearly 170 million confirmed cases of COVID19 worldwide with 3.5 million deaths due to or associated with SARS-CoV-2 disease. ${ }^{11}$ The worst affected countries at this point were the United States, India, Brazil, France, Russia, United Kingdom, Turkey, Italy, and Spain.

The COVID-19 pandemic has since the beginning of 2020 massively impacted patient safety - specifically health care associated infections and in particular nosocomial infections. The new disease and the pressing search for effective treatment strategies brought increased risk of both direct and indirect harm. ${ }^{1}$ The discomfort of personal protective equipment for health care workers (HCW) in operating rooms (OR), in COVID intensive care units (ICU), and on hospital wards, together with the attendant cumbersome and time-consuming donning, doffing, and hand hygiene procedures, have created new potential for hygiene failures on an enormous scale. ${ }^{6}$ Furthermore personal protective equipment hampers clear and unambiguous communication and exchange of medical information. Care in an ICU isolation bed leads to reduced social interaction and at least a partial reduction in surveillance by staff. Overall provision of care is disrupted by staffing shortages, work intensification, overtime, and staff redeployment. The physical and psychological well-being of HCWs becomes compromised with an ensuing negative effect on provision of stable and safe patient care.

Indirect effects of the pandemic on health care delivery have included delay in diagnostic procedures such as endoscopies or CT scans and the cancellation or postponement of elective but nonoptional operations. These decisions were made to mitigate risk of infection in both patients and HCWs and to free beds (and especially ICU beds) for COVID patients. In addition fear of nosocomial infection lead patients to delay hospital presentation in cases of emergent or persistent symptoms. This particularly endangered the safety of oncology patients, in whom preventable tumor growth and metastasis were special concerns. A reduction in diagnosis of early colon and lung cancers and an increase in first presentation at advanced stage of the same diseases was observed after the lockdowns of $2020 .{ }^{12}$ For surgeons a particular concern was risk of perioperative nosocomial COVID infection of their patients. This particular issue and other aspects of the impact of
COVID-19 infection on patient safety will be addressed quantitatively and on the basis of representative studies in the following sections.

\section{Perioperative COVID-19-Infection - Morbidity and Mortality}

First reports about unexpected fatality in the perioperative period among COVID-19-infected patients came from China. The mortality rate among a series of 34 patients undergoing surgeries (risk categories 1-4) during the incubation period of a COVID-19 infection was $20.5 \%$, whereas $44 \%$ of the patients in the same series required ICU care. $^{13}$

The COVID Surg Collaborative was very active in assembling timely data during 2020. They reported on 1128 patients with confirmed COVID-19 infection occurring between 7 days preoperatively and 30 days postoperatively ( $26 \%$ preoperative, $74 \%$ postoperative) from 235 hospitals in 24 countries. ${ }^{14}$ Fifty percent of these patients were 70 years or older and $74 \%$ had undergone emergency surgery. The total 30 -day mortality rate was $23.8 \%$, whereas $51.2 \%$ developed pulmonary complications. The subgroup of patients with severe acute perioperative respiratory syndromes had a 30-day mortality of $38 \%$ and accounted for $82 \%$ of all deaths. (Fig. 2) The rates of 30-day mortality and pulmonary complications after urologic, cardiac, vascular, or thoracic surgery $(30 \%-43 \%)$ were higher than those after gastrointestinal or orthopedic surgery $(23 \%-29 \%$ vs $44 \%-54 \%)$. Elective minor surgery (graded according to the Bupa schedule of procedures) in patients $<70$ years of age was associated with lowest risk whereas emergency major surgery (especially in those $>70$ years) was associated with highest risk. Emergency minor and elective major surgery was associated with similar outcomes.

Similar data were generated by a smaller Dutch study (Table 1) ${ }^{15}$ from 27 Dutch hospitals comparing the outcome of 161 patients with pre- or postoperative COVID-19 infection to outcome in a COVID-negative control group containing 342 patients. The 30-day mortality of the COVID positive group (16\%) was higher than in the controls $(4 \% P<0.001)$ as were the rates of pulmonary (24\% vs $3 \%, P<0.0001)$ and thromboembolic (7 vs $0.3 \% P<$ $0.0001)$ complications. After propensity matched scoring of 123 versus 196 patients the percentages were slightly lower but still showed the same significant differences. Both studies underline the risks of surgery and pulmonary complications in COVID-positive patients in general and especially older patients undergoing emergency surgery. The COVIDSurg study, published online in May 2020 , crucially informed both the decision of many stakeholders and

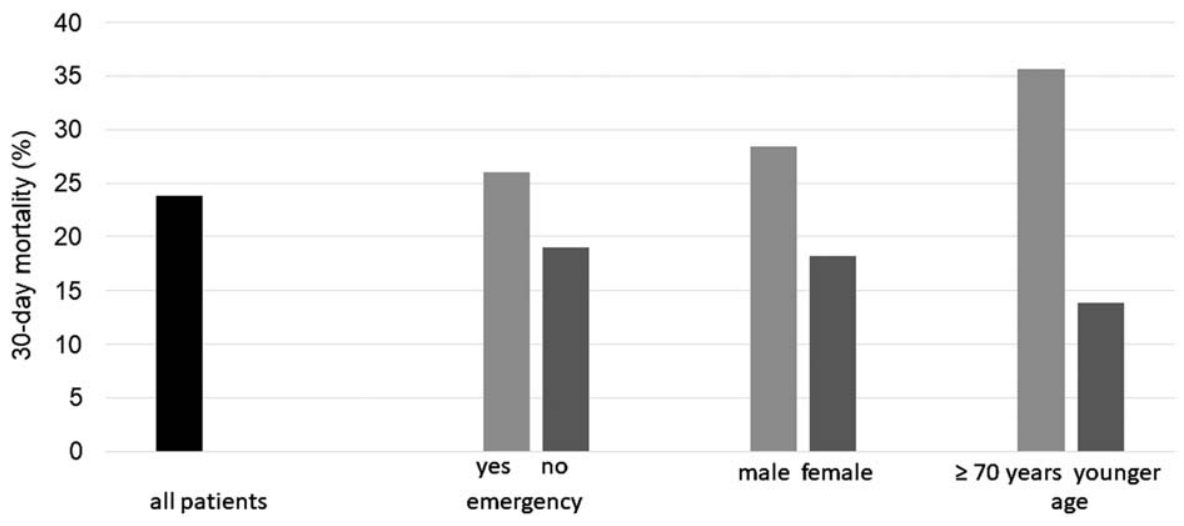

FIGURE 2. Thirty-day postoperative mortality of 1128 COVID-positive patients comparing emergency versus elective surgery, male versus female and age $\geq 70$ years or younger. ${ }^{14}$ 
TABLE 1. Dutch Study Comparing the Outcome of Consecutive Patients With Emergency or Elective Surgery and Preor Postoperative COVID-19 Infection to Outcome in a COVID-Negative Control Group. ${ }^{15}$

\begin{tabular}{lccc}
\hline & COVID-Positive & COVID-Negative & $\boldsymbol{P}$ \\
\hline Patients (n) & 161 & 342 & \\
Hospitals (n) & 27 & 4 & \\
30-Day mortality (\%) & 16 & 4 & $<0.001$ \\
Pulmonary & 24 & 3 & $<0.0001$ \\
$\quad$ complications (\%) & & 0.3 & $<0.0001$ \\
$\begin{array}{l}\text { Thromboembolic } \\
\quad \text { complications (\%) }\end{array}$ & 7 & & \\
\hline
\end{tabular}

medical associations to recommend restricting elective operations and the choice of many surgical departments to postpone operations during the early phase of the COVID-19 pandemic. ${ }^{14}$

Fortunately the rate of pulmonary complications in children with perioperative COVID-19 infection was low. An early report included a series of 88 children ( $64 \%$ boys) of 16 years or younger (from 52 hospitals in 21 countries) in whom a COVID-19 infection was proven between 7 days preoperatively (56\%) to 30 days postoperatively. ${ }^{16}$ Of these cases $89 \%$ involved emergency surgery; the surgical indications were benign disease $(81 \%)$, trauma $(11 \%)$, and cancer $(8 \%) .13 .6 \%$ of the children developed pulmonary complications defined as pneumonia, unexpected postoperative ventilation or ARDS. The 30-day mortality rate was only $1.1 \%(1 / 88)$, which compares very favorably to the mortality in high risk adults. ${ }^{14,15}$ Against this background it seems reasonable to suggest that the necessity of postponing pediatric operations during the COVID-19 pandemic can be discussed critically and to conclude that the management of provision of pediatric surgery during the pandemic may be subject to different principles than those concerning adult surgery. ${ }^{16}$

However recent reports of Multiple Inflammatory Syndrome in Children (MIS-C) several weeks after contracting the Corona virus is a cause of concern. ${ }^{17}$ Severe manifestations were primarily related to cardiac and/or pulmonary compromise. The seriousness of this new syndrome with regard to patient safety is illustrated by the reported clinical outcomes: $58 \%$ of 1733 MIC$\mathrm{C}$ children needed ICU-treatment and 24 (1.4\%) died. The postacute sequelae of SARS-CoV-2 infection ("long-COVID syndrome") is another danger for all patients who survive a nosocomial infection.

The risks associated with elective nonpalliative colorectal cancer surgery during the COVID-19 pandemic have been analyzed. ${ }^{18}$ In this series, cases of suspected or confirmed preoperative COVID infections were excluded. The collaborative reported on 2073 patients from 270 hospitals in 40 countries. A total of 947 of these operations involved the rectum and a stoma was placed in $34.2 \%$ of cases (including $4.3 \%$ so-called "COVID stomas" where conventional practice was changed to avoid possible complications requiring critical care). A postoperative COVID-19 infection was detected in $3.8 \%$ of these patients and an anastomotic leak rate of $4.9 \%$ was reported. The overall 30 -day mortality rate was $1.8 \%$. In patients without leak or without COVID the mortality rate was $0.9 \%$, whereas in patients with a combination of anastomotic leak and postoperative COVID infection the mortality rate was $38.5 \%$. The odds ratios of 30-day mortality were 6.0 for leak, 16.9 for postoperative COVID, 2.5 for males, 2.9 for age $>70$ years, and 3.4 for advanced cancer.
These findings represent the results of visceral cancer surgery during times of high COVID-19 incidence. Accordingly the most promising avenues for improving patient safety open to us are increasing anastomotic integrity and avoiding of nosocomial infection.

\section{COVID-19 Infection Risks Due to Aerosol Generated by Surgical Energy Devices}

The utilization of surgical energy devices during dissection produces smoke or plume with aerosolized particles of varying diameters. ${ }^{19,20}$ As a result there is a danger of inhalation of contaminated aerosol if the patient or staff is not sufficiently protected. Van Doremalen et al have shown that SARS-CoV-2 remains viable in an aerosol. $^{21}$ The largest particles come from ultrasonic shears and can be deposited in the oropharynx, trachea, bronchi, and bronchioli ${ }^{19}$; the smaller particles generated by laser and electrocautery can even reach the alveoli, whereas the diameter of the Corona virus (0.05$0.25 \mu \mathrm{m}$ ) is smaller than these electrocautery particles. Conventional surgical masks do not adequately protect against these particles; only particulate air respirator masks provide effective protection in the operating theatre environment. ${ }^{19}$

After analyzing the present literature on this issue the following can be concluded. 19,20,22-25 The transmission of virus via inhaled aerosol seems biologically plausible; no published research has identified COVID-19 particles within smoke from any surgical energy device in an operating room; conclusive evidence regarding the risk of COVID-19 infection via inhalation of bioaerosol is lacking.

Notwithstanding this the COVID-19-specific guidelines of numerous stakeholders and surgical associations recommend the following precautions in the OR: adequate Personal Protective Equipment (PPE), smoke extractors, air-filters, and OR airflow and specific operation theatre management. ${ }^{24,26,27}$

\section{Minimal Invasive Surgery During COVID-19 pandemic}

After the outbreak of COVID-19, a robust discussion began about the use of minimally invasive surgery (MIS). Most contentious was the aforementioned risk of contaminated bioaerosols. ${ }^{28}$ "Laparoscopy should generally not be used and considered, only in extremely selected cases" was the initial Intercollegiate General Surgery Guidance on COVID-19 from March 25, 2020 from four Royal Colleges and Associations in England, Scotland, and Ireland and the ACS. ${ }^{24,27,29,30}$ By April 2020, this initial strict warning had been qualified by the Australasian College, 3 Societies of Endoscopic Surgeons, and various authors ${ }^{22,27,30,31}$ : "Little or no evidence exists to favor an open approach over a laparoscopic/robotic one ${ }^{31}$; MIS offers significant advantages to both patient care and mitigation of the risk of viral transmission during surgery, provided the appropriate equipment and expertise are present" 27 ; "the advantages of Minimally Invasive Surgery should not be sacrificed in the current crisis. Prerequisites are strict theatre protocols, PPE, among others." 22

The initial strict advice of the Royal Colleges of Surgeons against MIS in March 2020 was subsequently modified and most surgeons now agree with the last 3 statements quoted above. There is now a consensus favoring MIS under defined protective preconditions. A study of nosocomial transmission rates showed that laparoscopic appendectomy was associated with a significantly reduced risk of perioperative COVID-19 infection and overall mortality when compared with open appendectomy. ${ }^{32}$ In the same study, MIS was also associated with a significantly reduced risk of perioperative COVID-19 infection when compared to open surgery among patients undergoing major upper GI or HPB procedures. 
After implementation of between case infection prevention and control procedures in the OR, the risk of symptomatic infection for patients who followed COVID-positive patients into the same OR was very low. ${ }^{33}$ After potential OR-related COVID exposure (ie, treatment in an OR which had been used for a COVID-positive case) within 24 hours $(n=23)$ or between 24 and 48 hours $(n=29)$ there were 0 cases of COVID-negative patients acquiring an infection.

\section{COVID-19 Free Surgical Pathways}

The effect of complete segregation of OR, ICU, and ward areas into COVID or non-COVID areas was studied in 9171 patients undergoing elective surgery for 10 solid cancer types in 447 hospitals in 55 countries. ${ }^{34}$ These did not include patients with a preoperative suspicion of COVID-19 disease. In the group of 6690 patients from "hot" hospitals with either incomplete COVID segregation or without defined COVID pathways, pulmonary complications were observed in $4.9 \%$ of cases and COVID-19 infections in $3.6 \%$. In the comparison group $(n=2481)$ of patients in COVID-19 free pathways there were pulmonary complications in $2.2 \%$ (OR 0.62 ) of cases and COVID-19 infections in 2.1\% (OR 0.53) of cases. Most endangered were the 238 patients from "hot" surgical units with postoperative COVID infections. Their pulmonary complication rate was $47 \%$ and mortality was $23 \%$.

A similar approach is that of COVID-19 free hospitals with centralization of COVID infected patients and separate surgical teams. A series of 198 patients in Ireland were screened for COVID-19; in 13 cases surgery was deferred because of symptoms or close contact to COVID-19; the remaining 185 COVID-negative patients underwent urgent operations in COVID free hospitals. ${ }^{35}$ COVID-related postoperative morbidity occurred in only 1 patient, who was immediately transferred. Reynolds from Dublin applied this pattern of management successfully to his patients with esophageal cancer in $2020(\mathrm{n}=45)$ and compared the results to his series in 2019 $(\mathrm{n}=53)$. The 90-day mortality and the perioperative COVID-19 infection rate in both years was $0 \% .{ }^{36}$ Results from a similar setting were reported for lung cancer surgery in the first 4 months of 2020 with $3 \%$ mortality in 734 patients (9 COVID-positive cases) and a single death related to COVID-19. ${ }^{37}$

\section{Medical Instead of Surgical Management During the COVID-19 Pandemic?}

In the early phase of the pandemic the ACS and the harmonized UK guidelines recommended medical management of acute issues that are typically treated operatively like appendicitis or cholecystitis/cholelithiasis. $^{27,29,30}$ The reasons for this were again concerns regarding perioperative Corona infection in both patients and staff. Two series of appendicitis patients before and after release of the UK guidelines were compared. ${ }^{38}$ The rate of nonoperative management increased significantly from $17 \%$ (11/63) preguidelines to $65 \%(51 / 79)$ post-guidelines. Relatedly laparoscopic approaches in operative cases dropped significantly from 94\% (49/52) to only $7 \%(2 / 28)$. The negative appendectomy rate remained unchanged and the Clavien-Dindo grade 3 to 5 rate was also unchanged (17\% vs $18 \%$ ). However, in the "post-guidelines" group of 28 appendicectomies one 80-year-old patient developed postoperative corona infection with respiratory failure and died. Six of the 51 "post-guideline" patients who had received medical care with antibiotic treatment had to be readmitted after discharge ( 2 requiring further antibiotics, 2 radiological drains, and 2 appendectomies).

If this COVID-related recommended change in conventional surgical practice persists or is indeed extended, a debate regarding the net effect on patient safety will have to occur, especially when considering the results of the prospective randomized APPAC trial. ${ }^{39}$
In this trial, $39 \%$ of 257 patients initially managed nonoperatively required appendectomy due to complications or recurrence within 5 years. A meta-analysis of 8 prospective studies showed that after antibiotic treatment of uncomplicated appendicitis the initial failure rate was $5 \%$ to $20 \%$ and the failure rate at 1 year after initial success was $10 \%$ to $37 \% .^{40}$ This means that about one-third of patients will need a second hospitalization with associated risk of COVID infection.

In Germany recommendations regarding the treatment of appendicitis did not change during the pandemic; nevertheless, the numbers of appendectomies did decrease. A register-based study involving 41 surgical departments in Germany compared February/ March 2019 to the same time period in 2020 (ie, during regional "lockdown" in Germany) and revealed a fall in appendectomies of $13.7 \%$ from 1027 to $888(P=0.003) .{ }^{41}$ The rate of complicated appendectomies increased from $58.2 \%$ to $64.4 \%$, whereas the percentage of negative appendectomies decreased from $6.7 \%$ to $4.6 \%(P$ $=0.012$ ). This can be interpreted as resulting from a combination of delayed presentation of symptomatic patients due to reluctance to seeking hospital care and from stricture patient selection for appendectomy. These changes have both potential disadvantages and advantages regarding patient safety. Of note postoperative morbidity or mortality and length of stay (LOS) did not increase in the 2020 cohort; only a small "high COVID-19 risk" subgroup ( $\geqq 70$ years, diabetes, immunosuppression, COPD) had more major postoperative complications $(12.5 \%$ vs $2.7 \% P<0.001)$ and needed more ICU treatment $(17.9 \%$ vs $2.3 \%, P<0.001)$ than the low-risk patients. Neither suspected nor proven COVID-19 infection had a significant effect on outcome.

Three further reports about the same period in Germany (early 2020) supported the aforementioned results and generated the following observations:

- The numbers of simple acute or nonacute appendicitis were most significantly reduced in females.

- The high rate of laparoscopic and low rate of nonoperative management of appendicitis did not change.

The general postoperative mortality of $0.2 \%$ after appendectomy was the same during the COVID pandemic when compared to previous years.

In March 2020, 4.8\% of patients with acute appendicitis had a concomitant COVID-19 infection. ${ }^{42-44}$

These results show that a shift to medical treatment of appendicitis or change in surgical technique due to the pandemic did not lead to clear evidence emerging of an improvement in patient safety.

\section{Cancellation / Postponement of Elective Surgery and Transplantation Due to COVID-19}

Indirect effects of the pandemic on patient safety were cancellation or postponement of elective operations. This was done to free beds (especially ICU beds) for potential COVID-19 patients, to prevent nosocomial COVID infections and to avoid exposure of OR personnel to infected patients. The resulting delay in treatment was especially serious for cancer patients because of the risks of preventable tumor growth and metastasis, or inappropriate timing of surgery following neoadjuvant therapy. ${ }^{45}$ Even a 4 -week delay in surgical treatment is associated with increased mortality in for example colorectal and lung cancer. ${ }^{46}$ For patients with benign diseases treatment delay results in prolongation of reduced quality of life combined with increased risk of an unfavorable course. According to a global expert response study from 190 countries, the total number of cancelled or postponed operations during the first 12 weeks of 
2020 were $28,404,603$; this represented 2,367,050 per week. ${ }^{47}$ The overall 12-week cancellation rate was $72 \%$ with the following distribution: $82 \%$ of benign operations (colorectal, upper GI, HPB together $81 \%$ ), $38 \%$ of cancer operations (colorectal 36\%, upper GI + HPB $40 \%$ ), and $25 \%$ of elective cesarean sections. The most cancellations were observed to have taken place in Europe and Central Asia.

The safety of a large number of patients in transplant programs was affected by COVID-19 mainly in two ways. First, the risk of viral infection in this group is by definition elevated, and the sequalae of infection when it occurs are more severe during both the pretransplant period for example in dialysis patients and the posttransplant period due to immunosuppression. ${ }^{48}$ Secondly limited access to transplantation is known to be associated with significant worsening of prognosis. ${ }^{24}$ Patients with pretransplant COVID infection were forced to accept prolonged waiting times. Living donor procedures were suspended during the first 6 months of the pandemic and the donor pool was further reduced by exclusion of COVID positive donors. ${ }^{49}$ COVID-19 caused particular problems in lung- and heart transplantation with a high mortality toll in cases of infection. Finally, the incidence of COVID-19 was high in procurement and transplant surgeons.

\section{SURGEON AND HEALTH CARE WORKER SAFETY}

\section{COVID-19 Infection Among Health Care Workers}

Doctors, nurses, and other health care workers (HCWs) who are employed in hospitals that treat patients with COVID-19 disease have a substantial risk of becoming infected and of all the attendant consequences of a severe or even fatal course. In an observational cohort study in the UK and the United States from March/April 2020 this risk was analyzed comparing front-line HCWs with individuals in the general community using a COVID symptom smartphone app (Table 2) ${ }^{50}$ The risk of COVID-19 infection was 12-fold higher in the HCW group. Front-line HCWs with adequate PPE caring for patients with suspected COVID-19 had a hazard ratio for COVID-19 infection of 2.4, whereas those treating patients with documented COVID-19 had a hazard ratio of 4.8 when compared to HCWs who were not involved in the treatment of COVID patients. In a hospital that was shut down due to a COVID-19 outbreak (COVID-19 positive rate of $35 \%$ among the 132 remaining patients) the risk of COVID infection for HCWs was 10- to 20-fold higher (and especially high in nurses) compared to the general population. ${ }^{51}$ COVID-19 risk was investigated in 28,792 Danish HCWs by screening for IgM and IgG serum antibodies against SARS-CoV-2 and comparing to blood donors. ${ }^{52}$ Seroprevalence was highest in HCWs on COVID-19 wards $(P<0.0001)$ followed by front-line HCWs $(P<0.0001)$. The seroprevalence of HCW not assigned to dedicated COVID-19 wards and that of blood donors was not significantly different. $53.5 \%$ of the 1163 seropositive HCW reported clinical symptoms attributable to COVID-19 disease.

To improve the safety of patients, doctors and nurses in the OR to ensure the completeness of appropriate measures against COVID infection, a modified surgical checklist has been proposed. ${ }^{53}$

TABLE 2. Prevalence of COVID-19 Infection Comparing $\mathrm{HCW}$ and General Individuals. ${ }^{50}$

\begin{tabular}{lcc}
\hline & $\begin{array}{c}\text { Front-Line } \\
\text { HCW }\end{array}$ & $\begin{array}{c}\text { Individuals From } \\
\text { General Community }\end{array}$ \\
\hline $\mathrm{N}$ & 99,795 & $2,035,385$ \\
COVID-19 infection per 100,000 & 2745 & 242 \\
\hline
\end{tabular}

Compared to the previous WHO-approved checklist the modified checklist contains additional items for all 3 time slots ("sign in," "time out," and "sign out") with questions on COVID typical symptoms, COVID testing, PPE, social distancing, and surgical energy devices (smoke extractors among others).

\section{Death of Health Care Workers by COVID-19 Infection}

Dr. Li Wenliang, a 34 year old ophthalmologist from Wuhan Central Hospital, was the first doctor to die due to a COVID-19 infection. ${ }^{54}$ His fate was particularly tragic because he was the first to notify the wider public of the emergence of SARS-CoV-2, having warned on December 30, 2019 on a Wechat Social Media Site about "seven cases of severe acute respiratory syndrome (SARS) illness with links to the Huanan Seafood Wholesale Market". He was reprimanded together with 7 others by security officers for "spreading rumors." Dr. Wenliang contracted COVID-19 and died despite intensive care treatment on February 7, 2020.

In the early phase of the COVID-19 pandemic (up to May 8, 2020) $152,888 \mathrm{HCW}$ were infected in 130 countries; this represented $3.9 \%$ of $3,912,156$ infected patients. ${ }^{55}$ The number of HCW deaths was 1413 from 67 countries. This means that nearly 1 in 100 COVID19 -infected HCWs died. This also amounts to $0.5 \%$ of 270,426 deaths from COVID infections worldwide. The median age and range of infected/deceased HCW was 47.3 (18-84) years/56.2 (18-84) years. Deceased HCW were much younger than patients who died of COVID in the general population (median 78 years). The highest case fatality rates were among 60- to 69-year olds (7\%) and $\geq 70$-year olds (37\%). Infections affected mainly females (72\%) and nurses $(39 \%)$, whereas deaths were mainly in males $(71 \%)$ and doctors $(51 \%)$. Concerning subspeciality assignment most reported deaths in nurses were in Internal Medicine and Mental Health and in doctors from Internal Medicine and General Practice; surgeons ranked third. The extent of the problem is presented in Table 3. ${ }^{55-59}$ Even more shocking are the published data from "The Guardian" newspaper that $>3600$ US-HCW died during the first year of the COVID pandemic. ${ }^{60}$ Amnesty International reported that at least 17,000 HCW have died worldwide from COVID-19 during 2020. ${ }^{61}$ Fortunately, vaccination of health care personnel appears to have substantially reduced COVID-19 infection and death rates of front-line workers in 2021.

\section{Mental Health of Surgeons During the COVID-19 Pandemic}

The increasingly stressful new working conditions of frontline staff and the dangers for HCWs of becoming personally infected or secondarily infecting their families have had a substantial psychological impact. ${ }^{62-65}$ The psychological impact of the pandemic on surgical staff was investigated in an international survey using a modified Delphi method. ${ }^{66}$ This study was based on 4282 responses from surgeons in 101 countries (60\% consultants, $46 \%$ general surgeons). Eighty-four percent cared for COVID-19 patients and $8.6 \%$ personally had had COVID-19. Thirty-two percent knew someone who died of COVID-19 (family members $4.5 \%$, colleagues/friends $27.5 \%$ ). For the subgroup who knew someone who died the percentage screening positive and the OR (parentheses) were as follows: depression $33 \%$ (1.3), anxiety $31 \%$ (1.6), stress $26 \%$ (1.4), and post-traumatic stress disorder $24 \%$ (1.7). The risk was greater for females and younger surgeons, those with less years of practice, and surgeons who worked closer to COVID-19 patients.

The mental health of surgical trainees was analyzed within a study on the impact of COVID-19 on surgical training. ${ }^{67}$ This systematic review included 29 articles with data from $>20$ countries 
TABLE 3. Reports of COVID-Infected/Deceased HCW in 2020 (Overlap Possible)

\begin{tabular}{|c|c|c|c|c|}
\hline Author & Origin & No. of Infected HCW & HCW Deaths & Case Fatality Rate \\
\hline Lapolla et $\mathrm{al}^{56}$ & Italy & 16.991 & 206 & $1.21 \%$ \\
\hline Bandyopadhyay et al $1^{55}$ & 130 Countries & 152.888 & 1413 & $0.92 \%$ \\
\hline Gholami et $\mathrm{al}^{57}$ & 7 Countries & 14.047 & 211 & $150 \%$ \\
\hline & & 58.473 (nurses) & 440 & $0.75 \%$ \\
\hline $\mathrm{CDCP}^{59}$ & USA (incl. 2021) & 381.524 & 1570 & $0.41 \%$ \\
\hline
\end{tabular}

with 5260 trainees and 339 program directors. A redeployment to nonsurgical roles varied across studies from $6 \%$ to $35 \%$ and operative experience had been reduced according to all studies. Negative associations with increased stress and reduced well-being were reported and ranged from 55\% to $97 \%$ of trainees. The reduced training opportunities and changed educational activities of surgical trainees during the pandemic may also have an impact on patient safety in the long run. ${ }^{6}$

\section{CONCLUSIONS}

Patient safety in surgery is an ongoing daily challenge and the SARS-2 Corona virus disease has brought additional risks of avoidable harm by infection during hospitalization. Compared to the prepandemic period patient safety is now inseparable from surgeon safety and that of all HCWs. The physical and psychological safety of all has been widely compromised by the COVID-19 pandemic. Returning to where we began with the painting of "Sciencia y Caridad" by Picasso, safety concerns once primarily centered on the patient are now also very relevant for the doctor and the nun at the patient's sides (Fig. 1). The safety of HCWs directly impacts on the safety of patients. It is for this reason that the World Health Organization dedicated the World Patient Safety day 2020 on September 17 to health care workers with the charter: "Health worker safety - a priority for patient safety." 69 We hope that by the time of publication of this editorial all measures against the COVID-19 pandemic and especially vaccination will have substantially improved the safety of patients, surgeons, and all $\mathrm{HCW}^{70}$ Nevertheless it is also important that we learn from history lessons of pandemics and from the tribulations in 2020 and 2021 and develop safe strategies to combat further pandemics of Corona virus mutants or indeed new viruses. ${ }^{71,72}$ As President of the ESA 2020 and 2021 I pay my deepest respects to all patients who suffered or died of COVID-19 infection during surgical treatment. The members of the ESA join with colleagues around the world in mourning the loss of numerous surgeons, doctors of cooperating disciplines, nurses, and other health care workers who became victims of the pandemic.

\section{REFERENCES}

1. WHO. Global Patient Safety Action Plan 2021-2030: Towards eliminating avoidable harm in 1. WHO. Global Patient Safety Action Plan 2021-2030: Towards eliminating avoidable harm in health care. World Health Organisation 2021.

2. Leriche R. La Philosophie de la Chirurgie., Vol. Chapitre 3. Paris: Flammarion \& Cie; 1951.

3. Slawomirski L, Auraeaen A, Klazinga N. The economics of patient safety: Strengthening a value based approach to reducing patient harm at national level 2017. Available at: https://doi.org/10.1787/5a9858cd-en.

4. Slawomirski L, Klazinga NS. Economies of patient safety: from analysis to action 2020. Available at: http://www.oecd.org/health-systems/Economics-ofPatient-Safety-October-2020.pdf.

5. WHO. Multi-professional Patient Safety Curriculum Guide. 2011.

6. WHO. Patient safety incident reporting and learning systems: technical report and guidance. Geneva: World Health Organization; 2020.
7. de Bienassis K, Kristensen S, Burtscher M, et al. Culture as a cure: assessments of patient safety Culture in OECD countries. In Developement OfECa, ed. OECD Health Working paper 119. Paris, 2020.

8. Clavien PA. Targeting quality in surgery. Ann Surg. 2013;258:659-668.

9. WHO. Patient Safety. Data and statistics [World Health Organization Regional Office for Europe website web site]. 2019. Available at: http://www.euro.who/ int/em/health-topics/Health-systems/patient-safety/data-and-statistics

10. Anesthesiology ESo, Association ES, al. e. Multi-disciplinary and patient centered approaches to perioperative patient safety. A European Consensus Statement. Patient Safety Policy Summit Brussels, 3-4 March 2020. Brussels, 2020.

11. WHO. WHO Coronavirus (COVID-19) Dashboard accessed 1st of May, 2021. Available at: https://covid19.who.int/.

12. Bertolaccini L, Sedda G, Spaggiari L. Paying another tribute to the COVID-19 pandemic: the decrease of early lung cancers. Ann Thorac Surg. 2021; 111:745-746

13. Lei S, Jiang F, Su W, et al. Clinical characteristics and outcomes of patients undergoing surgeries during the incubation period of COVID-19 infection. EClinicalMedicine. 2020;21:100331.

14. COVIDSurgCollaborative. Mortality and pulmonary complications in patients undergoing surgery with perioperative SARS-CoV-2 infection: an international cohort study. Lancet. 2020;396:27-38.

15. Jonker PKC, van der Plas WY, Steinkamp PJ, et al. Perioperative SARS-CoV-2 infections increase mortality, pulmonary complications, and thromboembolic events: a Dutch, multicenter, matched-cohort clinical study. Surgery. 2021; 169:264-274.

16. Nepogodiev D. Favourable perioperative outcomes for children with SARSCoV-2. Br J Surg. 2020;107:e644-e645.

17. Belay ED, Abrams J, Oster ME, et al. Trends in geographic and temporal distribution of US children with multisystem inflammatory syndrome during the COVID-19 pandemic. JAMA Pediatr. 2021.

18. Collaborative C. Outcomes from elective colorectal cancer surgery during the SARS-CoV-2 pandemic. Colorectal Dis. 2020.

19. Zakka K, Erridge S, Chidambaram S, et al. Electrocautery, diathermy, and surgical energy devices: are surgical teams at risk during the COVID-19 pandemic? Ann Surg. 2020;272:e257-e262.

20. Klompas M, Baker M, Rhee C. What is an aerosol-generating procedure? JAMA Surg. 2021;156:113-114.

21. van Doremalen N, Bushmaker T, Morris DH, et al. Aerosol and surface stability of SARS-CoV-2 as compared with SARS-CoV-1. N Engl J Med. 2020;382:1564-1567.

22. Mowbray NG, Ansell J, Horwood J, et al. Safe management of surgical smoke in the age of COVID-19. Br J Surg. 2020;107:1406-1413.

23. Zheng MH, Boni L, Fingerhut A. Minimally invasive surgery and the novel Coronavirus outbreak: lessons learned in China and Italy. Ann Surg. 2020;272:e5-e6.

24. Soreide K, Hallet J, Matthews JB, et al. Immediate and long-term impact of the COVID-19 pandemic on delivery of surgical services. Br J Surg. 2020; 107:1250-1261.

25. Perrone G, Giuffrida M, Bellini V, et al. Operating room setup: how to improve health care professionals safety during pandemic COVID-19-a quality improvement study. J Laparoendosc Adv Surg Tech A. 2021;31:85-89.

26. COVIDSurgCollaborative. Global guidance for surgical care during the COVID-19 pandemic. Br J Surg. 2020;107:1097-1103.

27. Chadi SA, Guidolin K, Caycedo-Marulanda A, et al. Current evidence for minimally invasive surgery during the COVID-19 pandemic and risk mitigation strategies: a narrative review. Ann Surg. 2020;272:e118-e124.

28. Bracale U, Podda M, Castiglioni S, et al. Changes in surgical behaviors during the CoviD-19 pandemic. The SICE CLOUD19 study. Updates Surg. 2021; $73: 731-744$ 
29. England RCoSo. Updated Intercollegiate General Surgery Guidelines on COVID-19. 2020. Available at: https://www.acpgbi.org.uk/content/uploads/ 2020/03/Updated-Intercollegiate-General-Surgery-Guidance-on-COVID-19final-with-logos13.pdf. Accessed March 25, 2020.

30. Surgeons ACo. COVID-19: Elective Case Triage Guidelines for Surgical Care. 2020. Available at: https://:www.facs.org/covid-19/clinical.guidance/elective.case. Accessed April 5, 2020, 2020.

31. SoAGaES, SAGES. SAGES and EAES Recommendations regarding surgical response to COVID-19 crisis 2020. Available at: https://www.sages.org/recommedations-surgical-response-Covid-19/. Accessed March 29, 2020, 2020.

32. Elliott JA, Kenyon R, Kelliher G, et al. Nosocomial SARS-CoV-2 transmission in postoperative infection and mortality: analysis of 14798 procedures. $\mathrm{Br} \mathrm{J}$ Surg. 2020;107:1708-1712.

33. Axiotakis LG Jr, Boyett DM, Youngerman BE, et al. SARS-COV-2 transmission rate is low when following a COVID+ patient in the operating room. Langenbecks Arch Surg. 2021;406:401-404.

34. Glasbey JC, Nepogodiev D, Simoes JFF, et al. Elective cancer surgery in COVID-19-free surgical pathways during the sars-cov-2 pandemic: an international, multicenter, comparative cohort study. J Clin Oncol. 2021;39:66-78.

35. Bolger JC, Kelly ME, Whelan C, et al. Public-private partnership: strategies for continuing urgent elective operative care during the COVID-19 pandemic. Br J Surg. 2020;107:e320-e321.

36. Bolger JC, Donlon NE, Butt W, et al. Successful maintenance of process and outcomes for oesophageal cancer surgery in Ireland during the first wave of the COVID-19 pandemic. Ir J Med Sci. 2021.

37. Seitlinger J, Wollbrett C, Mazzella A, et al. Safety and feasibility of thoracic malignancy surgery during the COVID-19 pandemic. Ann Thorac Surg. 2020.

38. English W, Habib Bedwani N, Smith C, et al. Suspected appendicitis and COVID-19, a change in investigation and management-a multicentre cohort study. Langenbecks Arch Surg. 2021;406:357-365.

39. Salminen P, Tuominen R, Paajanen H, et al. Five-year follow-up of antibiotic therapy for uncomplicated acute appendicitis in the APPAC randomized clinical trial. JAMA. 2018;320:1259-1265.

40. Collard M, Lakkis Z, Loriau J, et al. Antibiotics alone as an alternative to appendectomy for uncomplicated acute appendicitis in adults: changes in treatment modalities related to the COVID-19 health crisis. J Visc Surg. 2020;157(3s1):S33-S42.

41. Willms AG, Oldhafer KJ, Conze S, et al. Appendicitis during the COVID-19 lockdown: results of a multicenter analysis in Germany. Langenbecks Arch Surg. 2021;406:367-375.

42. Köhler F, Acar L, van den Berg A, et al. Impact of the COVID-19 pandemic on appendicitis treatment in Germany-a population-based analysis. Langenbecks Arch Surg. 2021;406:377-383.

43. Maneck M, Gunster C, Meyer HJ, et al. Influence of COVID-19 confinement measures on appendectomies in Germany-a claims data analysis of 9797 patients. Langenbecks Arch Surg. 2021;406:385-391.

44. Lock JF, Wiegering A. Changes in the management of acute appendicitis during the COVID-19 pandemic. Langenbecks Arch Surg. 2021;406:503-504.

45. Turaga KK, Girotra S. Are we harming cancer patients by delaying their cancer surgery during the COVID-19 pandemic? Ann Surg. 2020.

46. Hanna TP, King WD, Thibodeau S, et al. Mortality due to cancer treatment delay: systematic review and meta-analysis. BMJ. 2020;371:m4087.

47. COVIDSurgCollaborative. Elective surgery cancellations due to the COVID19 pandemic: global predictive modelling to inform surgical recovery plans. Br J Surg. 2020;107:1440-1449.

48. Michaels MG, La Hoz RM, Danziger-Isakov L, et al. Coronavirus disease 2019: implications of emerging infections for transplantation. Am J Transplant. 2020;20:1768-1772.

49. Kumar D, Manuel O, Natori Y, et al. COVID-19: a global transplant perspective on successfully navigating a pandemic. Am J Transplant. 2020;20:1773-1779.
50. Nguyen LH, Drew DA, Graham MS, et al. Risk of COVID-19 among frontline health-care workers and the general community: a prospective cohort study. Lancet Public Health. 2020;5:e475-e483.

51. Scherer MA, von Freyburg A, Brücher B, et al. COVID-19: SARS-CoV-2 susceptibility in healthcare workers-cluster study at a German teaching hospital. 4open. 2020;3.

52. Iversen K, Bundgaard H, Hasselbalch RB, et al. Risk of COVID-19 in healthcare workers in Denmark: an observational cohort study. Lancet Infect Dis. 2020;20:1401-1408.

53. Ambulkar RP, Singh P, Divatia J. Surgical safety checklist in the COVID era. $J$ Anaesthesiol Clin Pharmacol. 2020;36:289-290.

54. Petersen E, Hui D, Hamer DH, et al. Li Wenliang, a face to the frontline healthcare worker. The first doctor to notify the emergence of the SARS-CoV2, (COVID-19), outbreak. Int J Infect Dis. 2020;93:205-207.

55. Bandyopadhyay S, Baticulon RE, Kadhum M, et al. Infection and mortality of healthcare workers worldwide from COVID-19: a systematic review. BMJ Glob Health. 2020;5

56. Lapolla P, Mingoli A, Lee R. Deaths from COVID-19 in healthcare workers in Italy-What can we learn? Infect Control Hosp Epidemiol. 2021;42:364-365.

57. Gholami M, Fawad I, Shadan S, et al. COVID-19 and healthcare workers: a systematic review and meta-analysis. Int $J$ Infect Dis. 2021;104:335-346.

58. Erdem H, Lucey DR. Healthcare worker infections and deaths due to COVID19: a survey from 37 nations and a call for WHO to post national data on their website. Int J Infect Dis. 2021;102:239-241.

59. CDCP. COVID data tracker 2021. Available at: https://covid.cdc.gov/coviddata-tracker/\#cases_Accessed 4 May 2021, 2021.

60. [The Guardian web site]. 2021. Available at: https://www.theguardian.com/ theobserver/2021/apr/08.

61. Amnesty International. Mapping health worker deaths from COVID-19 2021. Available at: https://www.amnesty.org/en/latest/news/2020/09/mappingcovid19-health-worker-deaths/. Accessed March 5, 2021.

62. Torales J, O'Higgins M, Castaldelli-Maia JM, et al. The outbreak of COVID19 coronavirus and its impact on global mental health. Int J Soc Psychiatry. 2020;66:317-320.

63. Balasubramanian A, Paleri V, Bennett R, et al. Impact of COVID-19 on the mental health of surgeons and coping strategies. Head Neck. 2020;42:16381644.

64. Mehta S, Machado F, Kwizera A, et al. COVID-19: a heavy toll on health-care workers. Lancet Respir Med. 2021;9:226-228.

65. Pappa S, Ntella V, Giannakas T, et al. Prevalence of depression, anxiety, and insomnia among healthcare workers during the COVID-19 pandemic: a systematic review and meta-analysis. Brain Behav Immun. 2020;88:901-907.

66. Tan YQ, Wang Z, Yap QV, et al. Psychological health of surgeons in a time of COVID-19: a global survey. Ann Surg. 2021.

67. Hope C, Reilly JJ, Griffiths G, et al. The impact of COVID-19 on surgical training: a systematic review. Tech Coloproctol. 2021;25:505-520.

68. Daodu O, Panda N, Lopushinsky S, et al. COVID-19-considerations and implications for surgical learners. Ann Surg. 2020;272:e22-e23.

69. WHO. Charter Health Worker Safety: A Priority for Patient Safety. World Health Organisation; 2020.

70. Angel Y, Spitzer A, Henig O, et al. Association between vaccination with BNT162b2 and incidence of symptomatic and asymptomatic SARS-CoV-2 infections among health care workers. JAMA. 2021;325:2457-2465.

71. Straub KT, Jüni P, Urner M, et al. Public health interventions, epidemic growth, and regional variation of the 1918 influenza pandemic outbreak in a Swiss Canton and its greater regions. Ann Intern Med. 2021;174:533-539.

72. Mooney G. The dangers of ignoring history lessons during a pandemic. Ann Intern Med. 2021;174:556-557. 